\title{
2D-3D Synchronous/Asynchronous Camera Fusion for Visual Odometry
}

\author{
Danda Pani Paudel ${ }^{1}$ Cédric Demonceaux ${ }^{1}$ Adlane Habed $^{2}$ and Pascal Vasseur ${ }^{3}$
}

\begin{abstract}
We propose a robust and direct 2D-3D registration method for camera synchronization. Once the cameras are synchronized - or for synchronous setups - we also propose a visual odometry framework that benefits from both $2 D$ and $3 D$ acquisitions. Our method does not require a precise set of $2 \mathrm{D}$ to-3D correspondences, handles occlusions and works when the scene is only partially known. It is carried out through a $2 \mathrm{D}$ 3D based initial motion estimation followed by a constrained nonlinear optimization for motion refinement. The problems of occlusion and that of missing scene parts are handled by comparing the image-based reconstruction and 3D sensor measurements. The results of our experiments demonstrate that the proposed framework allows to obtain a good initial motion estimate and a significant improvement through refinement.
\end{abstract}

Index Terms-Asynchronous cameras, 2D-3D registration, Structure-from-Motion, Visual Odometry.

\section{INTRODUCTION}

The problem of accurately localizing cameras is of prime importance in many application involving visual Simultaneously Localization and Mapping (vSLAM). An accurate environment map is generally required for an accurate localization. In turn, building an accurate environment map is not possible without an accurate localization, hence, making it a paradoxical "chicken and egg" problem.

With the ongoing surge in affordable high quality 3D and 2D capture technologies, many mobile robots are, or can easily be, equipped with either or both vision modalities [1][19][22][23][24]. As far as 3D sensors are concerned, the Iterative Closest Point (ICP) algorithm (or one of its variants), applied on neighboring 3D point cloud measurements, is overwhelmingly used for robot localization. However, in the case of abrupt or long run displacements, localization based on 3D information alone is difficult mainly because of local minima traps (typical to ICP) and unreliable 3D feature descriptors. When a robot is equipped with both 3D and 2D sensors, 2D images are used to estimate the motion of the cameras (visual odometry) whereas the mapping is obtained directly from the 3D sensor. Indeed, the emergence of reliable 2D image feature descriptors (such as the ScaleInvariant Feature Transform (SIFT)), 2D-to-2D matching, generally supported by Random Sample Consensus (RANSAC), has become more reliable. However, the accuracy of the camera motion estimation from images, on which the robot localization relies, is undermined by the error amplitude of the extracted 2D features. When localization is based on 2Dto-3D correspondences and 2D-2D based refinement, it may

This research has been funded by an International Project NRF-ANR DrAACaR: ANR-11-ISO3-0003.

${ }^{1}$ Le2i UMR 6306, CNRS, University of Burgundy, France

${ }^{2}$ ICube UMR 7357, CNRS, University of Strasbourg, France

${ }^{3}$ LITIS EA 4108, University of Rouen, France suffer from significant error accumulation. One example of such error accumulation is shown in Fig. 11. This error is usually minimized by a loop closing technique as described in [20]. However, in particular when robots travel long distances, loop closing is not always possible and may not adequately compensate for error accumulation thus leaving visible artifacts in the map. Performing small and frequent loops are recommended as to keep the accumulated error under control. In practice, making such small loops while building large maps is undoubtedly a burden for the task at hand and often impossible. Though incorporating information from extra sensors such as GPS has been proposed [24][26], it is often argued that such information is neither accurate nor reliable enough.

Nowadays 3D sensors are providing increasingly high quality and accurate 3D measurements. Therefore, it has now become quite appealing and desirable to jointly benefit from the data acquired from both 2D and 3D modalities to achieve a better localization and/or motion estimation of the cameras at hand. Doing so accurately comes with its fair share of difficulties and challenges. Indeed, 2D and 3D camera setups generally require a full calibration of the system including 2D camera pose with respect to $3 \mathrm{D}$ measurements, i.e. extrinsic calibration, and synchronous acquisitions. Maintaining such a calibrated setup is both tedious and difficult due to possible changes in the camera pose parameters and the dedicated hardware required for synchronization. Note that changes in camera pose and/or the presence of synchronization delays, in particular in the case of fast moving systems, may result in large accumulated errors in the long run. Under such circumstances, or when the 3D and 2D captures are asynchronous, the 2D and 3D acquisitions need to be registered before they can be fused. While in the asynchronous case 2D-3D correspondences are unknown and need to be established, in the calibrated synchronous case, obtaining accurate 2D-to-3D matching is dependent upon the density of the 3D point cloud. Indeed, on the one hand, not every 3D point has known 2D corresponding points and, on the other hand, corresponding image points may not have the exact corresponding 3D point measurement present in the point cloud. Furthermore, whether the system is synchronous or not, some measurements captured by each modality may not be captured by the other. This mainly occurs because parts of the scene may be occluded by others. In the case of 3D captures, this results in (possibly large) missing parts from the scene. This renders the problem of registering data from both modalities rather challenging and difficult to solve.

In this paper, we propose a method for direct 2D-3D registration when $3 \mathrm{D}$ and $2 \mathrm{D}$ cameras are asynchronous. Once the asynchronous images are registered with the scene, they 
can be treated as synchronous acquisitions for which we propose a complete visual odometry framework that combines both $2 \mathrm{D}$ and $3 \mathrm{D}$ data. The proposed asynchronous 2D-3D registration method demands only a rough knowledge of the pose of only one of the cameras and, apart from 3D scene point coordinates, requires no other knowledge regarding the geometry of the input scene. We assume that point correspondences across images are available but 2D-to-3D correspondences are unknown. To our knowledge, there is no method that makes use of both 2D and 3D information without 2D-to-3D correspondences. Note that methods employing Bundle Adjustment (BA) with known scene [39] and PnP [18] require such 2Dto-3D correspondences to be established. In practice, good 2D correspondences between instantaneously captured images can be obtained by using state-of-the-art feature descriptors such as SIFT. The proposed method does not require a precise set of 2D-to-3D correspondences, handles occlusions, and works for partially known scenes. This framework computes the pose by localizing a set of cameras at once with respect to the $3 \mathrm{D}$ scene acquired in the previous frame using a minimum of three corresponding points among all the views. Furthermore, a constrained nonlinear optimization framework is also proposed for pose refinement. The first step of visual odometry uses only the known part of the scene whereas our refinement process uses the constraints that arise from the unknown part as well. The refinement step minimizes the projection errors of 3D points while enforcing the existing relationships between images. Both steps handle the problem of occlusion and that of missing scene parts by confronting the imagebased reconstruction and the $3 \mathrm{D}$ sensor measurements. They also minimize the effect of data inaccuracies by using an Mestimator based technique. Unlike [4], our method makes no prior assumption regarding the geometry of the scanned scene. Furthermore, the presented method differs from its preliminary works [41] [42] since it has been designed and tested for both synchronous and asynchronous cases in indoor as well as outdoor environments.

Our paper is organized as follows. Related work is presented in Section II The notations used in the present paper and the necessary background are introduced in Section III We formulate the optimization problem to obtain the optimal odometry parameters in Section IV. The solution to this problem is presented in the form of an algorithm in the same section. In Section V] experiments with synthetic and four real datasets are presented and discussed. Section VI concludes our work.

\section{RELATED WORK}

The 2D-3D registration problem is tackled in the literature through direct and indirect approaches. The direct registration methods rely on establishing feature correspondences (such as points, lines, planes, skylines and building bounding boxes) between the images and the 3D scene. The point-based matching methods proposed in [2], [3] require the 3D scene along with a scale invariant feature descriptor (SIFT) for each point. Correspondences are obtained by matching these feature descriptors to that of image feature points. Establishing reliable
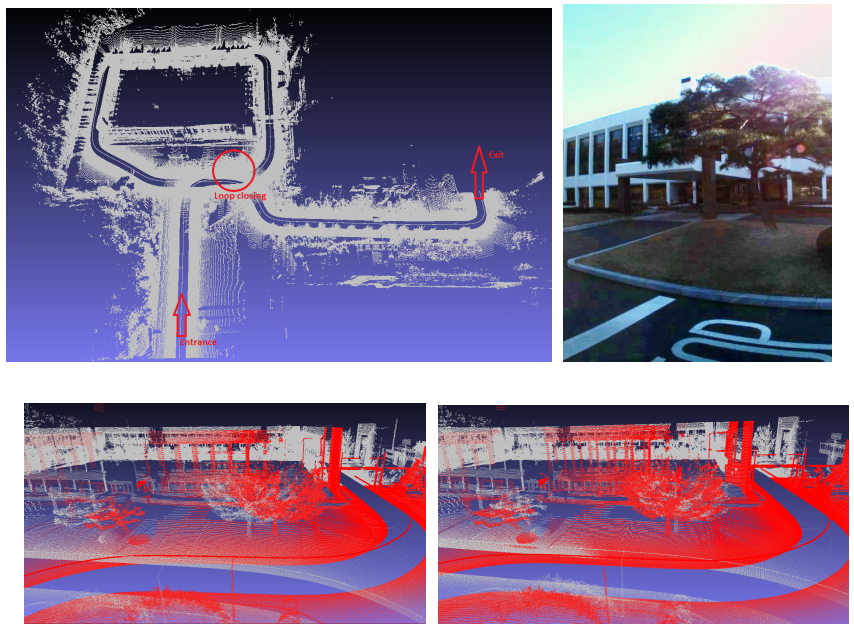

Fig. 1: An example of error accumulation around a loop: Map built by a Laser-Camera system around a large structure (top-left). Image taken at a loop closing point with only one tree at the corner (top-right). Map built before (red) and after (white) the visit around the loop using 2D-2D based refinement [24] (bottom-left). Refined map obtained using our method (bottom-right): the scan of the same tree come significantly closer after refinement.

correspondences may be undermined by the absence of such descriptors in the provided scene points as well as by the variability of the illumination conditions during the $2 \mathrm{D}$ and 3D acquisitions. Methods relying on higher level features, such as lines [5], planes [4] and building bounding boxes [7], are generally suitable for Manhattan World scenes (or the like) and hence applicable only in such environments. Skylinesbased methods [8] as well as methods relying on a predefined 3D model [12] are, likewise, of limited applicability. Indirect methods are performed either by 3D-3D registration or by finding some appropriate registration parameters. Methods based on 3D-3D registration are performed using the (rigid or non-rigid) Iterative Closest Point (ICP) algorithm between the Structure-from-Motion (SfM) induced reconstruction and the known scene. However, such registration is not straightforward due to the unknown scale of reconstruction. For instance, this scale ambiguity is handled by an extension of the 4-point congruent sets algorithm in [9]. On the other hand, registration based on complex parameters, such as mutual information [11] and region segmentation [10], are based on single images. Therefore, each camera requires its own initialization and is individually localized independently from the rest of the cameras. Cameras that are localized in this fashion may fail to satisfy the multiview geometric constraints (such as the epipolar constraint in two images).

Visual odometry is generally carried out by relying on 2D2D, 3D-3D, or 2D-3D information. 2D-2D based methods typically track features in monocular or stereo images and estimate the motion between them [28][29]. Some of these methods improve the localization accuracy by simultaneously processing multiple frames, while using BA for refinement. 
Some other methods obtain the motion parameters by registering images such that the photometric error between them is minimized [31], [32]. For the same purpose, most 3D-3D based methods use ICP or its variants [33][15][34] between consecutively acquired point clouds obtained from the 3D camera [38][37]. However, ICP-based methods are computationally expensive due to the calculation of the nearest neighbors for every point at each iteration. Both of these methods use the information from either camera only and, hence, do not fully exploit all the available information. Recent works [4][35] propose the use of information provided from both cameras during the process of localization. The work in [4] refines the camera pose obtained from Structure-fromMotion (SfM) using an extra constraint of a plane-induced homography via scene planes. This method provides a very good insight for a possibility to improve the camera pose when the partial 3D is known. However, it uses only the information from planes that are in the scene. The methods presented in [37][35][36] have been tested in indoor environments mainly with a Kinect sensor. Extension of these methods to outdoor environments with possibly different kinds of 3D cameras is not trivial due to various unhandled situations that may arise. Typical issues arising in outdoor scenes and/or different camera setups occur, for example, when 2D and 3D cameras do not share the exact same field of view, when the 3D points are sparse (as opposed to pixel-to-pixel mapping of RGB-D cameras), in the absence of required scene structures, and in the event of low frame rates and/or large displacements of the cameras. Note that other existing 2D-3D based refinement methods, such as BA and loop closing, are not applicable under these circumstances because they require precise 2D-to-3D correspondences across frames.

\section{NOTATION AND BACKGROUND}

The setup consists of a 3D scanner and multiple calibrated cameras as shown in Fig. 2. At any given instant, the 3D scanner scans the scene points $X_{k}, k=1 \ldots p$ in its coordinate frame $O^{1}$. A set of calibrated cameras at $R_{i} \mid t_{i}, i=1 \ldots m$, not necessarily overlapping, capture $m$ images, from which a set of 2D feature points are extracted. Let $x_{i j}^{1}, j=1 \ldots n$ represent those feature points in the $i^{t h}$ image. $P(R, t, X)$ is the projection function that maps a point $X$ to its $2 \mathrm{D}$ counterpart in the image captured from $R \mid t$. When the system moves by $R^{\prime} \mid t^{\prime}$ to next position, corresponding variables are represented by the same notations with change in superscript. The poses of the second set of cameras with respect to $O^{1}$ are expressed as $\overline{R_{i}} \mid \overline{t_{i}}$. The Essential matrix between two views of the same camera in different frames is expressed as

$$
E_{i}\left(R^{\prime}, t^{\prime}\right)=\left[t_{i}^{\prime}\right]_{\times} R_{i}^{\prime},
$$

where $R_{i}^{\prime} \mid t_{i}^{\prime}$ is the pose of $i^{t h}$ camera in the second frame with respect to the first one. For synchronous setups, it is related to $R^{\prime} \mid t^{\prime}$ as follows

$$
\left(\begin{array}{cc}
R_{i}^{\prime} & t_{i}^{\prime} \\
0 & 1
\end{array}\right)=\left(\begin{array}{cc}
R_{i} & t_{i} \\
0 & 1
\end{array}\right)\left(\begin{array}{cc}
R^{\prime} & t^{\prime} \\
0 & 1
\end{array}\right)\left(\begin{array}{cc}
R_{i} & t_{i} \\
0 & 1
\end{array}\right)^{-1} .
$$

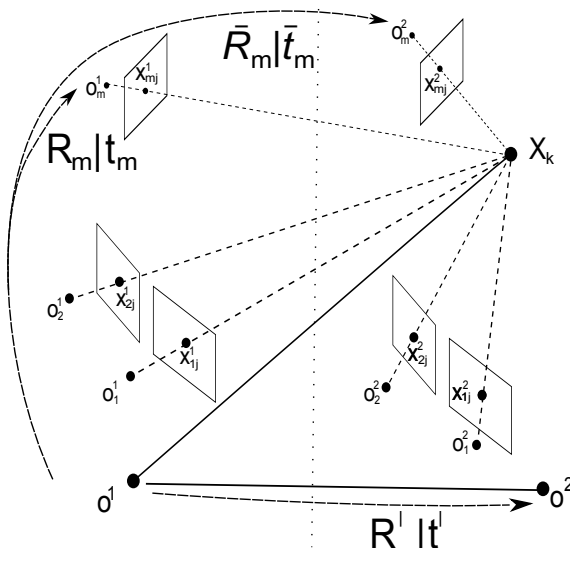

Fig. 2: Ray diagram of the experimental setup.

If $x_{i j}^{1}$ and $x_{i j}^{2}, j=1 \ldots n$ are corresponding feature points in two consecutive images taken by the $i^{t h}$ camera, their 2Dto-3D correspondences are specified by a function $\phi$. Let $\phi_{i}(j)$ be a function that maps each pair of 2D points $x_{i j}^{1} \leftrightarrow x_{i j}^{2}$ to the corresponding 3D point $X_{k}$. Every rotation matrix $R$ is represented by a $4 \times 1$ vector of quaternions $q$ unless mentioned otherwise (similarly, $q^{\prime}$ for $R^{\prime}$ ). Both 3D and 2D points are represented by $3 \times 1$ vectors, the latter being the homogeneous representation in the camera coordinate system. The distance between two rotation matrices is measured by computing the spectral norm of their difference. For a matrix $A$, its spectral norm is denoted as \|\|$A\|\mid\|$. Two given up-to-scale translation vectors are compared by measuring the angle between them.

\section{2D-3D VISUAL ODOMETRY}

In this section, we establish the relationships between a set of image pairs and scene points. Using these relationships, we propose an optimization framework whose optimal solution is the required odometry parameters. A complete algorithm for solving this optimization problem is also discussed. The proposed method deals with both the asynchronous and synchronous cases separately. In the asynchronous case, the camera's extrinsic parameters $R_{i}^{\prime} \mid t_{i}^{\prime}$ are assumed to be unknown. In the synchronous case these parameters are known and fully exploited during the motion estimation process. We also assume that the 2D-to-2D correspondences between image pairs acquired by the same camera are known.

\section{A. Problem formulation}

The relationship between 2D and 3D points is depicted in the ray diagram given in Fig. 2. The projection error of points on the first set of cameras is given by

$$
e^{1}\left(R_{i}, t_{i}, \phi_{i}(j)\right)=\left\|x_{i j}^{1}-P\left(R_{i}, t_{i}, X_{\phi_{i}(j)}\right)\right\|^{2} .
$$

Similarly, for the second set of cameras, the projection error us given by

$e^{2}\left(R_{i}, R^{\prime}, t_{i}, t^{\prime}, \phi_{i}(j)\right)=\left\|x_{i j}^{2}-P\left(R_{i} R^{\prime}, R^{\prime} t_{i}+t^{\prime}, X_{\phi_{i}(j)}\right)\right\|^{2}$. 
Furthermore, the epipolar constraint that relates the points in two views of different frames can be written as

$$
\left(x_{i j}^{2}\right)^{T} E_{i}\left(R^{\prime}, t^{\prime}\right) x_{i j}^{1}=0 .
$$

While (3) locates the first camera, (4) locates the second camera with respect to the world reference frame while preserving its relationship to the first one. Similarly, (5) localizes the second camera with respect to the first one. Equations (3), (4) and (5) are obviously redundant. However, in the presence of noise in the data and unknown correspondences all constraints must be enforced: satisfying only the non-redundant conditions does not necessarily satisfy all of them. In addition, (5) makes use of the unknown part of the scene as well. Therefore, all three equations will be incorporated in our optimization framework in which (3) is chosen to be the objective (as it includes the pose of both the cameras) while the rest are used as constraints.

Our problem is to localize a set of $2 \mathrm{D}$ cameras with known 2D-to-2D $\left(x_{i j}^{1} \leftrightarrow x_{i j}^{2}\right)$ and unknown 2D-2D-to-3D $\left(x_{i j}^{1} \leftrightarrow x_{i j}^{2} \leftrightarrow X_{\phi_{i}(j)}\right)$ correspondences in the presence of noise. Hence, finding the optimal $\phi_{i}$ itself is part of the optimization process. Therefore, the optimization framework can be written as

$$
\begin{aligned}
& \min _{q_{i}, t_{i}, q^{\prime}, t^{\prime}, \phi} \sum_{i=1}^{m} \sum_{j=1}^{n}\left\|x_{i j}^{2}-P\left(R_{i} R^{\prime}, R^{\prime} t_{i}+t^{\prime}, X_{\phi_{i}(j)}\right)\right\|^{2}, \\
& \text { subject to }\left\|x_{i j}^{1}-P\left(R_{i}, t_{i}, X_{\phi_{i}(j)}\right)\right\|^{2}=0 \\
&\left(x_{i j}^{2}\right)^{T} E_{i}\left(R^{\prime}, t^{\prime}\right) x_{i j}^{1}=0 \\
&\left\|q_{i}\right\|^{2}=1,\left\|q^{\prime}\right\|^{2}=1, i=1 \ldots m, j=1 \ldots n .
\end{aligned}
$$

The optimization problem (6) considers that every image point has its corresponding 3D point in the scene. In practice, there could be extra $2 \mathrm{D}$ or missing $3 \mathrm{D}$ points resulting in invalid 2D-to-3D correspondences. We address this problems by assigning the weights derived from a scale histogram to each correspondence.

If $\widetilde{X}_{i j}$ is the two-view reconstruction, the relative scale of reconstruction for known 3D-to-3D correspondences $\widetilde{X}_{i j} \leftrightarrow$ $X_{\phi_{i}(j)}$ is computed as

$$
s_{i}(j)=\frac{\left\|R_{i}^{T} \widetilde{X}_{i j}-R_{i}^{T} t_{i}\right\|}{\left\|X_{\phi_{i}(j)}\right\|}, \quad j=1 \ldots m .
$$

Since the reconstructed points from each pair share a common scale, in the ideal case, we have $s_{i}(j)=$ const $_{i} . \forall j \in 1 \ldots n$. In practice, when the histograms $H_{i}(u), u=1 \ldots b$ of these scales are built, they hold the highest number of samples in the bin corresponding to the true scale. If those bins are $U_{i}$, then the weights are distributed as follows:

$$
w_{i}(j)= \begin{cases}1 & s_{i}(j) \in H\left(U_{i}\right) \\ 0 & \text { otherwise }\end{cases}
$$

Furthermore, the effect of data inaccuracies is reduced by introducing a robust estimation technique. Hence, the optimization problem (6) with robust estimation and histogrambased weighting can be re-written as

\begin{tabular}{|c|c|c|}
\hline & Input & Estimation \\
\hline \hline & 2D-2D corresp. & $R_{i}, t_{i}$ and $\bar{R}_{i}, \bar{t}_{i}$ \\
\hline Asynchronous & 2D-2D corresp., $R_{i}, t_{i}$ & $R^{\prime}, t^{\prime}$ \\
\hline Synchronous & 2D
\end{tabular}

TABLE I: Known and estimated parameters.

$$
\min _{\substack{q_{i}, t_{i}, q^{\prime}, t^{\prime}, \phi}} \sum_{i=1}^{m} \sum_{j=1}^{n} w_{i}(j) \rho\left(\left\|x_{i j}^{2}-P\left(R_{i} R^{\prime}, R^{\prime} t_{i}+t^{\prime}, X_{\phi_{i}(j)}\right)\right\|\right),
$$

subject to $\rho\left(\left\|x_{i j}^{1}-P\left(R_{i}, t_{i}, X_{\phi_{i}(j)}\right)\right\|\right)=0$,

$$
\rho\left(\left(x_{i j}^{2}\right)^{T} E_{i}\left(R^{\prime}, t^{\prime}\right) x_{i j}^{1}\right)=0
$$$$
\left\|q_{i}\right\|^{2}=1,\left\|q^{\prime}\right\|^{2}=1, i=1 \ldots m, j=1 \ldots n .
$$

where $\rho(x)$ is Tukey bi-weighted potential function. For a threshold $\xi$, it is defined as

$$
\rho(y)= \begin{cases}\frac{y^{6}}{6}-\frac{\xi^{2} y^{4}}{2}+\frac{\xi^{4} y^{2}}{2} & \text { for }|y|<\xi \\ \frac{\xi^{6}}{6} & \text { otherwise }\end{cases}
$$

whose influence function is $\psi(y)=y\left(\xi^{2}-y^{2}\right)^{2}$ for $|y|<\xi$ and 0 otherwise.

Note that any 2D-to-3D correspondence that does not vote for the valid scale is considered to be an outlier. Here, the derived cost depends only upon the known part of the scene whereas the constraint includes the unknown part as well. The optimal odometry parameters are obtained by iteratively solving this optimization problem. Each iteration breaks the problem down into two subproblems: (a) 2D-to-3D registration and (b) Camera pose refinement.

\section{B. $2 D$-to-3D registration}

The registration step coarsely localizes the cameras with respect to the scene. Here, we discuss the registration methods for asynchronous and synchronous cases as two separate subproblems. In the asynchronous case, finding the 2D-to3D correspondences required for registration is not trivial. This is done by iterating between camera poses and the correspondence estimation. On the other hand, finding the precise cross-frame correspondences for the synchronous case is not easy either. Cross-frame image-to-scene registration in synchronous acquisition is carried out by using minimal point RANSAC-based pose estimation. The choice of registration methods depends upon the experimental setup. The known input and estimated parameters for two different cases are summarized in the Table [

1) Asynchronous case: The main problem in the asynchronous acquisition is that the poses of the camera with respect the scene are unknown. This makes solving 2D-to3D correspondence problem very challenging. Since these correspondences are unknown, the reconstruction that can be obtained from images is related to the scene by an unknown scale factor. To avoid the role of this unknown scale, we minimize a cost function which is independent of it, while imposing the epipolar constraint between images. The proposed optimization problem for asynchronous cameras registration is as follows: 


$$
\min _{\substack{q_{i}, t_{i} \\ \phi}} \sum_{i=1}^{m} \sum_{j=1}^{n} w_{i}(j) \rho\left(\left\|\left(x_{i j}^{2}\right)^{T} E_{i}\left(R^{\prime}, t^{\prime}\right) P\left(R_{i}, t_{i}, X_{\phi_{i}(j)}\right)\right\|\right),
$$

subject to $w_{i}(j) \rho\left(\left\|x_{i j}^{1}-P\left(R_{i}, t_{i}, X_{\phi_{i}(j)}\right)\right\|\right)=0$,

$$
\left\|q_{i}\right\|^{2}=1 . i=1 \ldots m, j=1 \ldots n \text {. }
$$

The initial estimate of $R_{i}^{\prime}, t_{i}^{\prime}$ is obtained using the SfMbased relative pose estimation method. In this case, we choose $\phi$ such that it maps every pair of image points to a 3D point that respects the constraint while minimizing the cost. The constraint violation is penalized by a simple but effective static penalty function as discussed in [21]. Therefore,

$$
\begin{aligned}
\phi_{i}(j) & =\underset{k \in\{1, \ldots, p\}}{\operatorname{argmin}}\left\|x_{i j}^{1}-P\left(R_{i}, t_{i}, X_{k}\right)\right\| \\
& +\left\|\left(x_{i j}^{2}\right)^{T} E_{i}\left(R^{\prime}, t^{\prime}\right) P\left(R_{i}, t_{i}, X_{k}\right)\right\|, j=1 \ldots n .
\end{aligned}
$$

Hence, the optimal poses of the first set of cameras are

$$
\underset{q_{i}, t_{i}}{\operatorname{argmin}} \sum_{i=1}^{m} \sum_{j=1}^{n} w_{i}(j) \rho\left(\left\|\left(x_{i j}^{2}\right)^{T} E_{i}\left(R^{\prime}, t^{\prime}\right) P\left(R_{i}, t_{i}, X_{\phi_{i}(j)}^{1}\right)\right\|\right)
$$

subject to $w_{i}(j) \rho\left(\left\|x_{i j}^{1}-P\left(R_{i}, t_{i}, X_{\phi_{i}(j)}\right)\right\|\right)=0$,

$$
\left\|q_{i}\right\|^{2}=1 . i=1 \ldots m, j=1 \ldots n \text {. }
$$

This is a constrained nonlinear optimization problem on the quaternion parameters whose local optimal solution can be obtained by the iteratively re-weighted least-squares (ILRS) technique. In fact, depending upon one's choice, it can also be solved linearly on $R$ and $t$ using singular value decomposition. However, the linear solution does not constrain $R$ to be a rotation matrix. Therefore, the obtained solution needs to be enforced as a rotation matrix before extracting the quaternion parameters.

For each pair of images, the scale of the reconstruction is finally estimated by averaging the scales of inliers as follows

$$
\mu_{i}=\frac{\sum_{j=1}^{n} w_{i}(j) s_{i}(j)}{\sum_{j=1}^{n} w_{i}(j)}, \quad i=1 \ldots n .
$$

Finally, the absolute poses of the second set of cameras in $O^{1}$ can be obtained through

$$
\left(\begin{array}{cc}
\overline{R_{i}} & \overline{t_{i}} \\
0 & 1
\end{array}\right)=\left(\begin{array}{cc}
R_{i}^{\prime} & \mu_{i} t_{i}^{\prime} \\
0 & 1
\end{array}\right)\left(\begin{array}{cc}
R_{i} & t_{i} \\
0 & 1
\end{array}\right) .
$$

Once the cameras are fully registered, they can be thought as synchronized ones. This is because the second set of cameras can be localized in the first coordinate frame. Henceforth, we assume that the unsynchronized cameras are synchronized once registered to the scene.

2) Synchronous Case: It is trivial to find the 2D-to-3D correspondences $X_{k} \leftrightarrow P\left(R_{m}, t_{m}, X_{k}\right)$ in one frame. However, cross-frame correspondences are required in order to estimate the motion $R^{\prime} \mid t^{\prime}$. Such correspondences can be obtained by matching the $2 \mathrm{D}$ feature points between images. Note that most $P\left(R_{m}, t_{m}, X_{k}\right)$, when considered as feature points, are unlikely to result in reliable feature descriptors for matching. Therefore, we extract a separate set of $2 \mathrm{D}$ feature points to obtain better 2D-2D correspondences $x_{i j}^{1} \leftrightarrow x_{i j}^{2}$. Methods based on relative pose require at least 5 such correspondences to compute the motion with an unknown scale. On the other hand, if 2D-to-3D correspondences $x_{i j}^{2} \leftrightarrow X_{k}$ can be found, it would require only 3 points to estimate the motion including the scale. In order to benefit from this, the required 2D-to$3 \mathrm{D}$ correspondences are computed for each image which is established by the mapping function $\phi_{i}(j)$ computed as

$$
\phi_{i}(j)=\underset{k \in\{1, \ldots, p\}}{\operatorname{argmin}}\left\|x_{i j}^{1}-P\left(R_{i}, t_{i}, X_{k}\right)\right\|, j=1 \ldots n .
$$

It is important to notice that the correspondences obtained in this manner are not perfect. We make a strong consideration of this restriction while refining the estimated motion. The search required to minimize (16) can be performed using a KD-tree like structure where the projections of all 3D points build one tree in each image. The detected feature points traverse these trees in search for the best possible match. Once the required correspondences are obtained, the set of cameras in the second frame can be localized with respect to previously acquired 3D scene using the method presented in [25]. The advantage of using this method is that it requires a 'minimum of 3 correspondences among all the views and does not require a complex scene as demanded by ICP or SfM. For example, even a planar scene with sufficient texture can be processed. For low frame rates and/or large displacements, feature matching methods still work better than tracking them. Since only 3 correspondences are needed, finding them from already matched $2 \mathrm{D}-2 \mathrm{D}$ to sparse $3 \mathrm{D}$ is very much achievable in practice.

\section{Camera pose refinement}

Recall that in both asynchronous and synchronous cases the final result is the registration of next frame images to the previous scene. In fact, the obtained registration parameters are the absolute poses of the cameras. However, in practice, the motion obtained in this manner is not very accurate. In this step, we refine these coarse motion/registration parameters while making use of scene information. The refinement process optimizes the motion parameters such that the SfM reconstruction is the closest to the known scene. During this process, the asynchronous setups are refined by directly solving the equation presented in (9) for the known correspondence function $\phi$. The correspondences required in this step are obtained directly from the registration process. However, the synchronous setups are refined by solving the following optimization problem:

$$
\begin{aligned}
& \min _{q^{\prime}, t^{\prime}} \sum_{i=1}^{m} \sum_{j=1}^{n} w_{i}(j) \rho\left(\left\|x_{i j}^{2}-P\left(R_{i} R^{\prime}, R^{\prime} t_{i}+t^{\prime}, X_{\phi_{i}(j)}\right)\right\|\right), \\
& \text { subject to } \rho\left(\left(x_{i j}^{2}\right)^{T} E_{i}\left(R^{\prime}, t^{\prime}\right) x_{i j}^{1}\right)=0, \\
& \quad\left\|q^{\prime}\right\|^{2}=1, i=1 \ldots m, j=1 \ldots n .
\end{aligned}
$$

This is again a constrained nonlinear optimization problem that can be solved by ILRS technique. Each iteration of IRLS uses the interior-point method to solve the constrained nonlinear least-squares problem. 


\section{The algorithm}

Starting from known 2D-to-2D correspondences, the algorithm iteratively estimates the odometry parameters mentioned in Table I. Every iteration reduces the cost function (9) in two steps while satisfying its constraints. Here, we present two different algorithm for asynchronous and synchronous cases separately.

$\overline{\text { Algorithm } 1 \text { Asynchronous case }}$
For known initial guess on $R_{i} \mid t_{i}$ and $R_{i}^{\prime} \mid t_{i}^{\prime}$ obtained from
relative pose estimation, refine them through the following two
steps:

1) Camera alignment: iteratively align the cameras to scene until convergence,

a) estimate the relative pose using $2 \mathrm{D}$-to-2D correspondences;

b) compute 2D-to-3D correspondences using (12);

c) build multiple scale histogram $H_{i}(u)$ and compute weights $w_{i}(j), j=1 \ldots n$;

d) update the pose of the first set of cameras using (13).

2) Simultaneous pose refinement: starting from the results obtained in the "Camera alignment" step, refine poses of both sets of cameras by solving (9).

Obtain real scale $\mu_{i}$ and compute the absolute pose using (15).

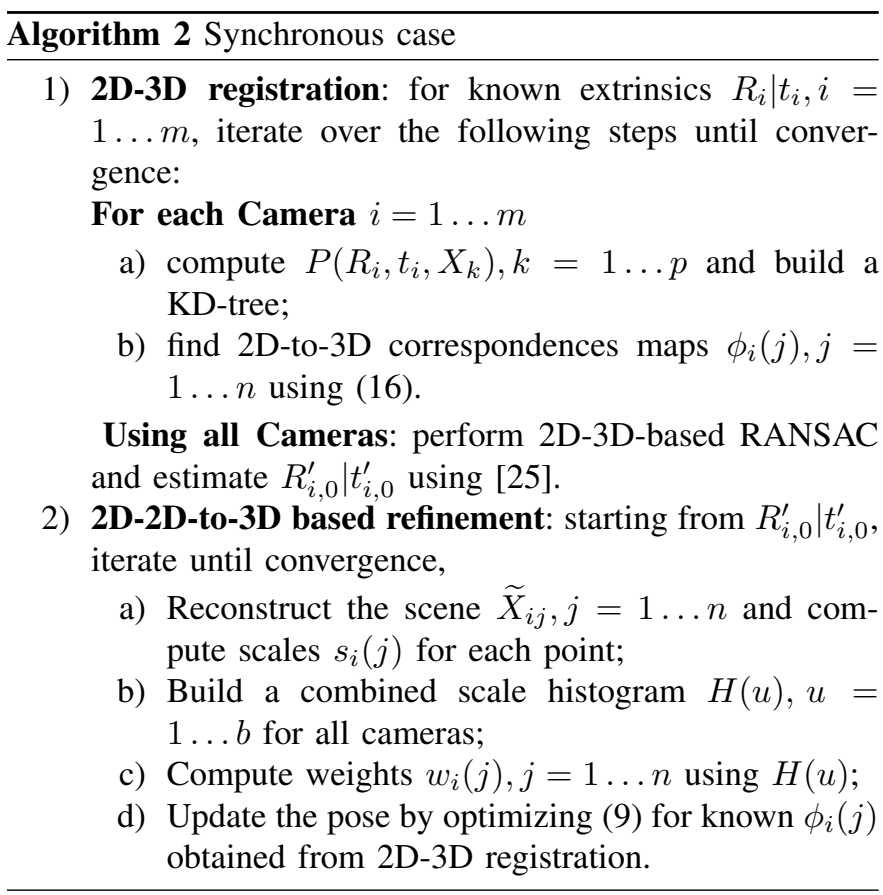

\section{E. Normalization and pose recovery}

For the sake of numerical stability, the 3D scene points are normalized such that the distance between the scene's centroid to the first camera is approximately equal to 1 . If the initial estimate of the first camera pose is $\left\{R_{i, 0}, t_{i, 0}\right\}$, such normalization corresponds to $\widehat{X^{i}}=\left(R_{0, i} X+t_{0, i}\right) / \| t_{0, i}||, i=$
$1 \ldots m$. After this transformation, $R_{i, 0}$ and $t_{0, i}$ simplify to $I_{3 \times 3}$ and $0_{3 \times 1}$ respectively. We also normalize the data during the robust estimation i.e. $y$ in Equation (10) is scaled with twice of its median value and $\xi$ is set to 1 whenever it is used. The iterations are terminated when the improvement of the pose between two consecutive iterations $k-1$ and $k$ of both cameras becomes insignificant. The improvements on the rotational $(R)$ and translational $(t)$ components are computed using

$$
e_{R}=||\left|R_{k}-R_{k-1}\right||| \text { and } e_{t}=\cos ^{-1}\left(\frac{t_{k}^{T} t_{k-1}}{\left\|t_{k}|||| t_{k-1}\right\|}\right) \text {. }
$$

Improvements on $R^{\prime}$ and $t^{\prime}$ are also computed similarly. The algorithm terminates when $e_{R}<T_{1}, e_{R^{\prime}}<T_{1}, e_{t}<T_{2}$, and $e_{t^{\prime}}<T_{2}$ for some given thresholds $T_{1}$ and $T_{2}$.

\section{EXPERIMENTS}

We tested our methods using both synthetic and real datasets. Our results with synthetic data were compared against those of ICP with classical SfM. For real data, experiments with four different datasets captured under different setups were performed. In all the cases, the constrained nonlinear least-squares optimization problem was solved by using MATLAB-R2012a Optimization Toolbox with interior-point method.

\section{A. Simulations}

We generated a set of 800 random 3D points scattered on the surface of four faces of a $\left[\begin{array}{lll}-10 & 10\end{array}\right]^{3}$ cube. The cameras were placed about $20 \pm 2$ units away from the origin with randomly generated rotations while roughly looking towards the centroid of the scene. All scene points were projected onto $256 \times 256$ images with zero-skew, 100 pix. focal length and an image-centered principal point. The $2 \mathrm{D}$ data were obtained by adding various levels of zero-mean Gaussian noise to the pixel coordinates. 400 out of 800 projected points were randomly selected and used to localize the second camera with respect to the first one using classical SfM. During this process, half of the points are rejected to minimize the effect of outliers thus leading to the reconstruction of only 200 points. The same data were used in our method to perform the registration and the refinement. We ran 100 tests for each noise level of standard deviation from 0 to 2.0 with a 0.25 step. The simulation results are presented for the two-view case only.

The roughly known $R$ was generated by introducing an error of $[0.050 .075]^{c}$ in roll, pitch and yaw each. We introduced these relatively small errors in $R$ to observe the improvement when the iterative scheme converges. Similarly, a small error of $\pm 5 \%$ was introduced in each translation axis. Nevertheless, these errors are very significant since the scene is relatively far from the cameras. The histogram was built with auto adjustable 10 bins after discarding the scales of less than 0.1 and greater than twice its median. First, we obtained the best possible $R, t, R^{\prime}$, and $t^{\prime}$ using classical SfM [30] and ICP[16]. As ICP cannot be performed without the knowledge of relative scale, the extra information of scale is recovered with the assumption of the image-based reconstruction being 

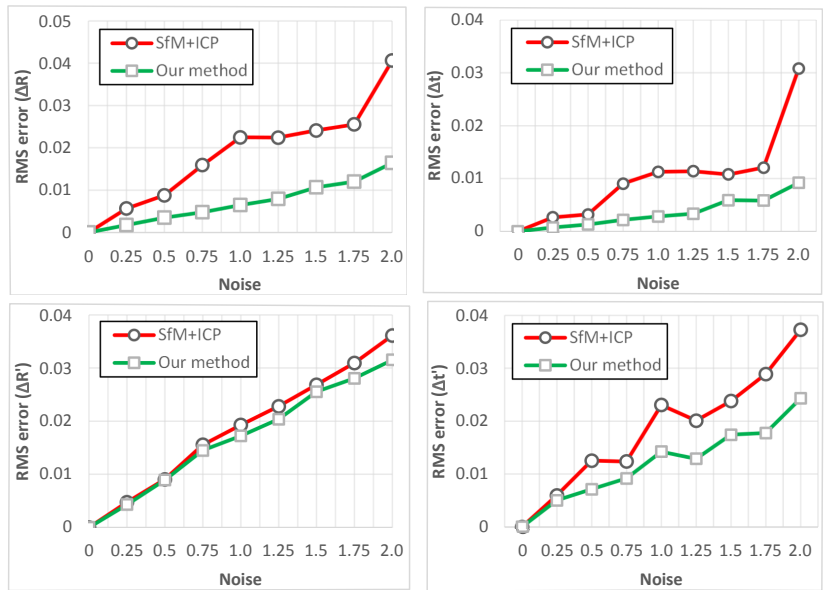

Fig. 3: SfM+ICP vs. Our method with noise; $\Delta R$ (left-top), $\Delta t$ (right-top), $\Delta R^{\prime}$ (left-bottom), and $\Delta t^{\prime}$ (right-bottom).

spread all over the provided 3D scene. Note that, our method does not require this extra information of scale. To analyze the improvement on camera pose, we computed the deviation of these results from their ground truth values. The errors $\Delta R$, $\Delta t, \Delta R^{\prime}$, and $\Delta t^{\prime}$ correspond to the residuals computed as in (18). Fig. 3 shows the Root-Mean Square (RMS) plots of the computed errors for various levels of noise. It can be seen that our method performs significantly better than SfM with ICP even when the ICP is favored with extra information of scale.

\section{B. Real data}

Three benchmark and one in-house real datasets were used to test the proposed algorithms. Two out of these four datasets were acquired asynchronously and the other two synchronously. Each of these datasets were acquired by very different setups as discussed below. The results obtained were compared against the ground truth (whenever available) or the known desired output. Required 2D-to-2D correspondences for all the experiments were obtained by the SURF descriptor based matching.

1) Asynchronous case: Scene and images were captured by two different devices. The first dataset was captured by a Kinect sensor and a separate 2D camera. The second dataset consists of two different scenes scanned by a laser-scanner and multiple images captured by a camera. Results for the second dataset were compared against the provided groundtruth values. However, the results of the first dataset were compared against the desired reconstruction.

Kinect Dataset: For the first experiment with real data, we built the prior 3D scene by registering multiple frames acquired from a 3D sensor (Kinect). This scene was then down-sampled to about 50,000 points as shown in Fig. 4 (left). After the 3D scene is acquired, a standard-sized football was placed in the same scene and two $1080 \times 1920$ images were captured by a moving camera. These images and their 1198 correspondences are shown in Fig. 4 and Fig. 5. 14 manually selected points from the corners of the Truncated Icosahedron (TI) (Fig. 5 (right)) were retained for assessing

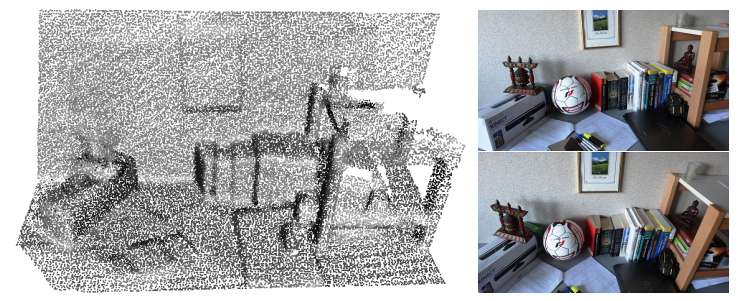

Fig. 4: Left: Kinect 3D scene; Right: image pair.
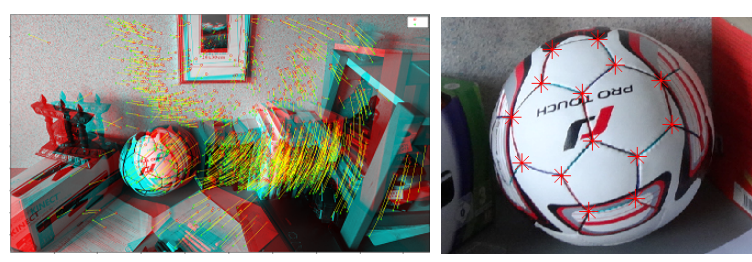

Fig. 5: Left: Correspondences; Right: feature points.

the quality of the reconstruction. To overcome the problem of initialization, the first views of both $2 \mathrm{D}$ and 3D cameras are captured approximately from the same location while facing towards the same part of the scene.

The final metric reconstruction of the scene is upgraded to Euclidean for the measured length of polygon sides equal to $4.5 \mathrm{~cm}$. Reconstructed TI from two views is placed in the given 3D scene and shown in Fig. 6. We have approximated the circumference of the football by fitting a sphere passing through the vertices of the reconstructed TI. For a quantitative analysis, the following geometric parameters of reconstructed TI are computed: (i) LS: RMS error of the length of sides. (ii) AH: RMS error of the internal angles of hexagons. (iii) AP: RMS error of the internal angles of pentagons. (iv) AHP: RMS error of Dihedral angles between hexagons and the pentagons. (v) A-HH: Dihedral angle between two hexagons (expected: 138.19). (vi) CS: Circumference of the sphere (expected: $68-70 \mathrm{~cm}$ ). Table II] compares these parameters against FIFA's standard. This is an example of 2D-to-3D data fusion where the reconstruction from two views is added to the 3D scene. This example also demonstrates the handling of occlusion problem because of the football placed in the scene after the 3D acquisition. Furthermore, even when the 3D data is not very accurate, as it is the case in this case, it shows that our method still benefits from the scene information.

EPFL dataset: We also tested our method with the public datasets Fountain-P11 and Herz-Jesu-K7 (Fig. 7 from http: //cvlabwww.epfl.ch/ $\sim$ strecha). These datasets consist, respectively, of 11 and 7 images of size $3072 \times 2048$ along with
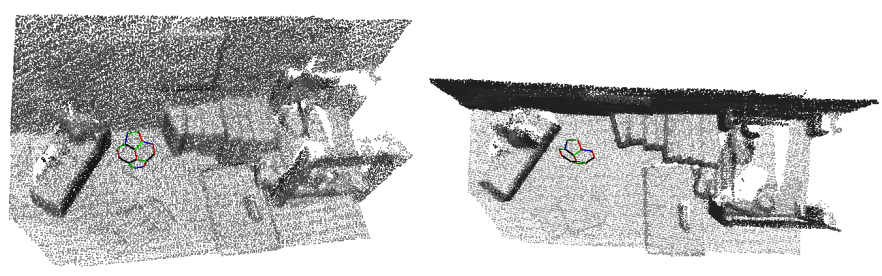

Fig. 6: Two views of the 3D scene with TI. 


\begin{tabular}{|c|c|c|c|c|c|c|}
\hline & $\begin{array}{c}\text { LS } \\
(\mathrm{cm})\end{array}$ & AP & AH & A-HP & A-HH & $\begin{array}{c}\text { CS } \\
(\mathrm{cm})\end{array}$ \\
\hline \hline SfM & 0.201 & 4.267 & 2.008 & 6.195 & 140.19 & 76.25 \\
\hline Our method & 0.117 & 2.943 & 0.863 & 3.342 & 139.20 & 73.10 \\
\hline
\end{tabular}

TABLE II: Geometric parameters.
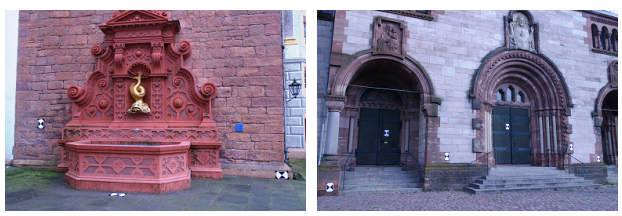

Fig. 7: Left: Fountain-P11; Right: Herz-Jesu-K7.

ground truth partial 3D point clouds of the scenes. To validate the ground truth, the texture was mapped on the scene by back-projecting images using their ground truth projection matrices. Fig. 8 shows that the provided camera poses are very satisfactory (unlike M. Corsini et al. reported in [9]). First, the 3D reconstructions for every consecutive pair of images are obtained using classical SfM. All these results are then refined separately using our method. Results before and after the refinement are compared against the ground truth in Table III The 3D errors shown here are the mean 3D RMS error of all the pairs. During the implementation, we have decimated the 3D scenes to about 50,000 points by uniform down-sampling for a faster computation. About 2000-3000 feature points were selected in each pair of views for the reconstruction.

For the multiview case, reconstructions from each consecutive pair of views are registered. Such registration undergoes error accumulation and scale factor drift. We separately refined these results using our method and sparse BA [27]. The results using our method were found to be significantly better than those of BA. We also considered refining our results using BA. Results obtained from BA, our method, and BA performed to refine our results are shown in Table IV] It is observed that BA performed on our results diverges from the ground truth instead of further refinement. Since BA takes only the image information into account and cannot incorporate the $3 \mathrm{D}$

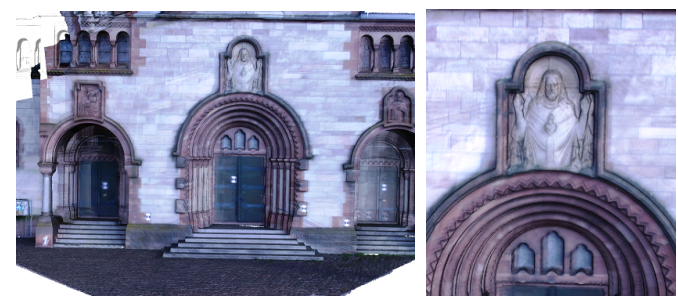

Fig. 8: Texture mapping of Herz-Jesu-K7.

\begin{tabular}{|c|c|c|c|}
\hline & Method & Fountain & Herz-Jesu \\
\hline \hline \multirow{2}{*}{$\Delta R^{\prime}$ (RMS) } & SfM & 0.0044 & 0.0072 \\
\cline { 2 - 4 } & Our method & $8.49 \mathrm{e}-4$ & 0.0013 \\
\hline \multirow{2}{*}{$\Delta t^{\prime}$ (RMS) } & SfM & 0.0404 & 0.0757 \\
\cline { 2 - 4 } & Our method & 0.0031 & 0.0052 \\
\hline \multirow{2}{*}{$3 \mathrm{D}$ error } & SfM & 0.0011 & 0.0025 \\
\cline { 2 - 4 } & Our method & $5.95 \mathrm{e}-4$ & 0.0018 \\
\hline
\end{tabular}

TABLE III: SfM vs. our method (two views).

\begin{tabular}{|c|c|c|c|}
\hline & Method & Fountain & Herz-Jesu \\
\hline \hline \multirow{3}{*}{$\Delta R^{\prime}$ (RMS) } & BA & 0.0436 & 0.0123 \\
\cline { 2 - 4 } & Our method & 0.0020 & 0.0067 \\
\cline { 2 - 4 } & Refined & 0.0251 & 0.0080 \\
\hline \multirow{3}{*}{$\Delta t^{\prime}$ (RMS) } & BA & 0.0311 & 0.0402 \\
\cline { 2 - 4 } & Our method & 0.0019 & 0.0224 \\
\cline { 2 - 4 } 3D error & Refined & 0.0172 & 0.0241 \\
\hline & BA & 0.0020 & 0.0069 \\
\cline { 2 - 4 } & Our method & 0.0015 & 0.0068 \\
\cline { 2 - 4 } & Refined & 0.0020 & 0.0069 \\
\hline
\end{tabular}

TABLE IV: BA vs. Our method and unsuccessful refinement of our results using Bundle Adjustment - BA (multiview).
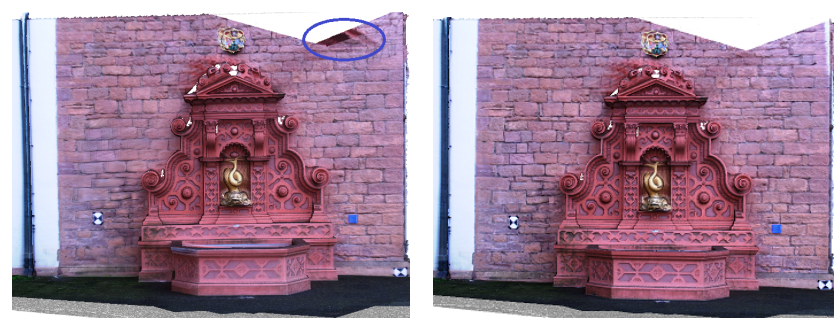

Fig. 9: Texture mapping: Bundle Adjustment (left), our method (right).

knowledge, noise present in the image might be the reason for BA to diverge. For qualitative analysis, results obtained from BA as well as our method were used to map the texture (Fig. 9). Texture mapping using BA contains many artifacts the most visible of which has been circled in this figure. Note that, as the scene being relatively far from the cameras, even a small error in pose can significantly affect the texture mapping. It clearly shows the pose refinement using our method is very accurate and visually no different from the ground truth.

2) Synchronous case: We have also tested our method using two different real and synchronous datasets. Both datasets were acquired by a moving vehicle equipped with a lasercamera system. However, these two setups greatly differ from one another.

KAIST Dataset: We conducted our first Synchronous experiment using data obtained from a Laser-Camera system dedicated to reconstructing very large outdoor structures. This system uses two 2D laser scanners and four 2D cameras which are synchronized and calibrated for both intrinsic and extrinsic parameters. Laser scanners used here provide a wide angle of view of the scanning plane so that the system can observe tall objects as well as the ground making its suitable to scan the environment from a close distance. The 3D map (reconstruction) of the environment is made by collecting these 2D scans at their proper location. Therefore, this system requires a very precise localization for a good reconstruction. Extrinsic parameters of 2D cameras were estimated by laser points and a pattern-based calibration method. However, it still possesses a mean projection error of about 0.5 pixels. The interested reader may refer to [24] for details regarding the experimental setup. The dataset we have tested is a continuous trip of the Laser-Camera scanning system within the compound of KAIST (Korea) for a distance of about $3 \mathrm{KM}$. The system made seven different loops during its travel. The original reconstruction and the loops are shown in Fig. 10 


\begin{tabular}{|c|c|c|c|}
\hline Loop & Size $(\mathrm{m})$ & Bok et al. $(\mathrm{m})$ & Our method $(\mathrm{m})$ \\
\hline \hline 1 & 351.76 & 4.063 & 1.548 \\
\hline 2 & 386.38 & 4.538 & 1.469 \\
\hline 3 & 224.37 & 4.765 & 4.398 \\
\hline 4 & 242.87 & 1.696 & 1.077 \\
\hline 5 & 931.14 & 3.884 & 2.858 \\
\hline 6 & 1496.4 & 7.182 & 6.381 \\
\hline 7 & 546.05 & 5.502 & 2.115 \\
\hline
\end{tabular}

TABLE V: Loop size and loop closing errors in meters for Bok et al. [24] and our method.

The lengths of the loops, as shown in Table $\mathrm{V}$, range from about 200 meters to $1.5 \mathrm{KM}$. Each camera captured $480 \times 640$ pix. images with a rate of about 20 frames/sec. The 2D-to2D correspondences are computed between images escaping each 10 frames. The original reconstruction obtained by the Laser-Camera system was used as the required 3D information for our method. Note that this reconstruction was not very accurate. Nevertheless, we were still able to refine the motion using such inaccurate data.

The qualitative and quantitative results are presented in Fig. 11 and Table $\mathrm{V}$ respectively. The errors were computed by performing the ICP between two point clouds captured at the loop closing point before and after the loop travel. Note that loop closing methods are not applied to the presented results. Our goal is to obtain a better localization so that it would be suitable for the loop closing methods. We strongly believe that the localization with such accuracy can be a very suitable input for loop closing. Our experiments clearly show significant improvement in loop closing errors by our method for all the loops tested. Since, most of the loop closing methods used in practice provide only the local optimal solution; these improvements contribute to their convergence to the desired one. It can also be seen that the error reduction is independent of the loop length. In fact, the improvement is dependent upon the quality of feature points. The remaining residual error is the combined effect of the errors in calibration, matching, and measurements.

To analyze reconstruction accuracy, we fitted the surface on the reconstructed points cloud using an algorithm that we have developed in-house. This algorithm takes advantage of the camera motion and the order of scanned points. The reconstructed surface was mapped with texture from the same images that were used for localization. The textured scene with its various stages is shown in Fig. 12 for only one side of the reconstruction around the first loop (about 350 meters). This part of the reconstruction consists of about $1.3 \times 10^{6} 3 \mathrm{D}$ points and $2.5 \times 10^{6}$ triangles.

KITTI Dataset: The proposed method was also tested on the benchmark dataset available at (http://www.cvlibs.net/datasets/kitti/). The details of the experimental setup is described in [40]. We have used the stereo pair of gray images and the 3D data scanned from a Velodyne laser scanner. The results obtained before and after refinement for 5 different sequences were compared against the provided ground truth. Errors in rotation and translation were computed by using the evaluation code provided along with the dataset which uses the ground truth obtained using
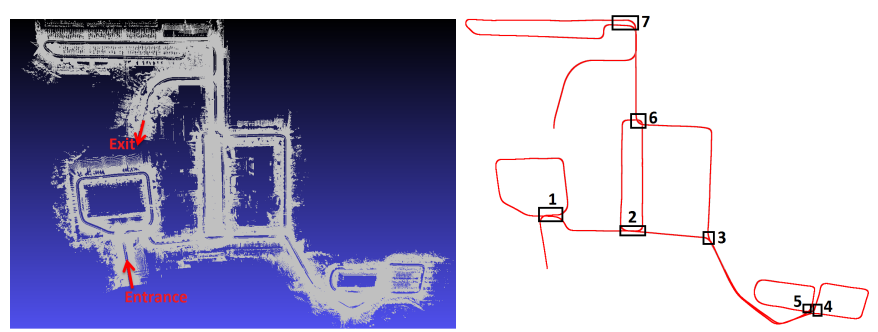

Fig. 10: Large map reconstructed using Laser-Camera system in a single trip shown with starting and end points (left). Closed loops made during the travel. Boxes shown are the loop closing locations of seven different loops (right).
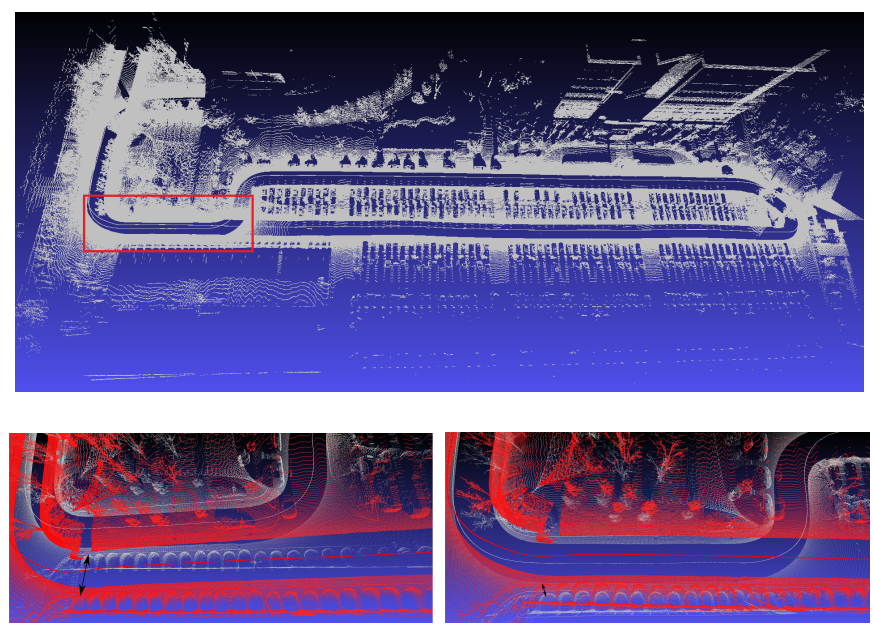

Fig. 11: Results similar to Fig. 1 for seventh Loop. Reconstruction with a red box at the loop closing location (top), obtained using Bok et al. (bottom-left) and our method after refinement (bottom-right). The double sided arrows show the gap between two different reconstructions of the same scene.

GPS and other odometry sensors. Although this ground truth might not be very accurate for local poses comparison, it is relevant over a long sequence due to no error accumulation process. Therefore, the errors were measured at the sequence steps of $(100,200, \ldots, 800)$ and are presented in Table VI Fig. 13 shows the map obtained for the fifth sequence. A close observation shows that the localization before the refinement is already quite satisfactory. Its further refinement makes the result very close to the ground truth itself. Here again, the results are presented without the loop closing.

\begin{tabular}{|c|c|c|c|c|c|}
\hline \multirow{2}{*}{ Sq.N } & \multirow{2}{*}{ N.Frames } & \multicolumn{2}{|c|}{ Initial Estimate } & \multicolumn{2}{c|}{ Refined } \\
\cline { 3 - 6 } & & $\Delta T(\%)$ & $\Delta R(\% / m)$ & $\Delta T(\%)$ & $\Delta R\left({ }^{\circ} / m\right)$ \\
\hline \hline 3 & 801 & 1.6774 & 0.000432 & 1.6398 & 0.000216 \\
\hline 5 & 2761 & 1.9147 & 0.000245 & 1.8679 & 0.000162 \\
\hline 7 & 1101 & 2.3410 & 0.000231 & 1.5689 & 0.000192 \\
\hline 8 & 4071 & 2.3122 & 0.000447 & 1.9799 & 0.000196 \\
\hline 9 & 1591 & 1.7562 & 0.000270 & 1.5604 & 0.000197 \\
\hline
\end{tabular}

TABLE VI: Translation $(\Delta T)$ and Rotation $(\Delta R)$ errors in Initial and Refined results for five different sequences. 

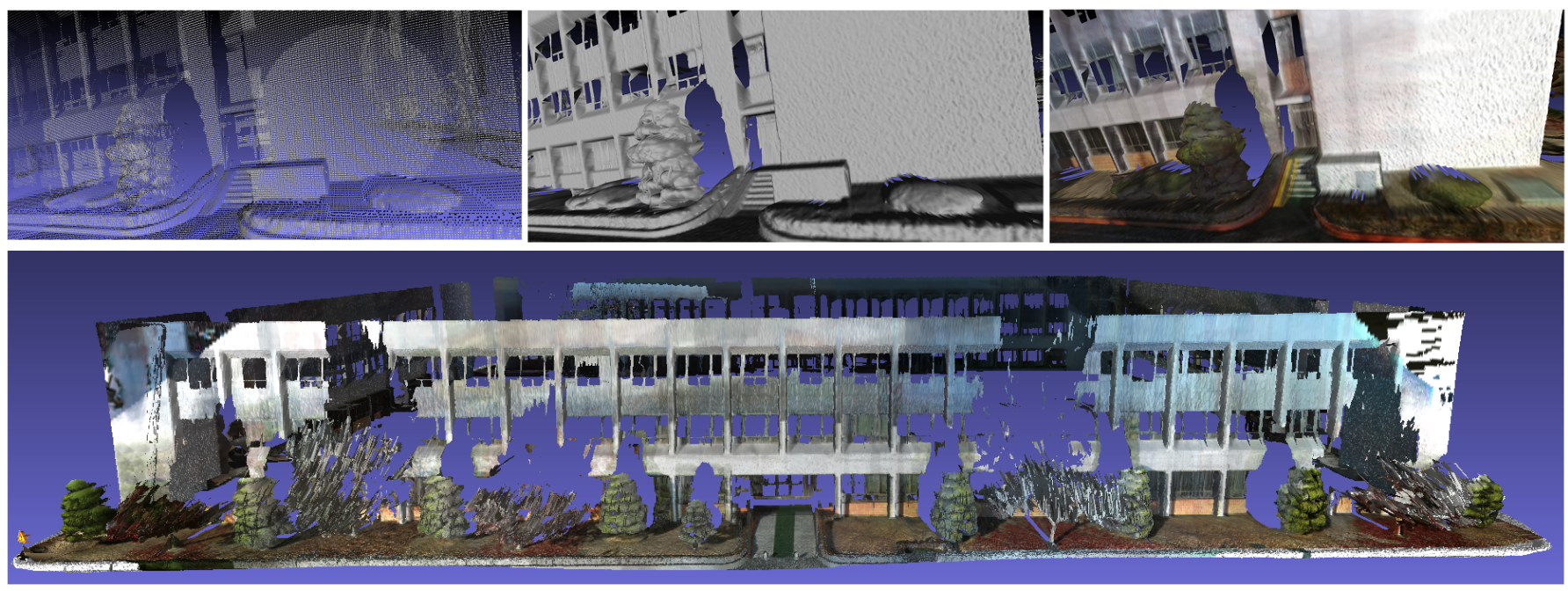

Fig. 12: Surface reconstruction and texture mapping showing the accuracy of localization. Reconstructed 3D, fitted surface, and texture mapping in a close view (top row, left to right). Texture mapping of the structure scanned around loop 1 (bottom).

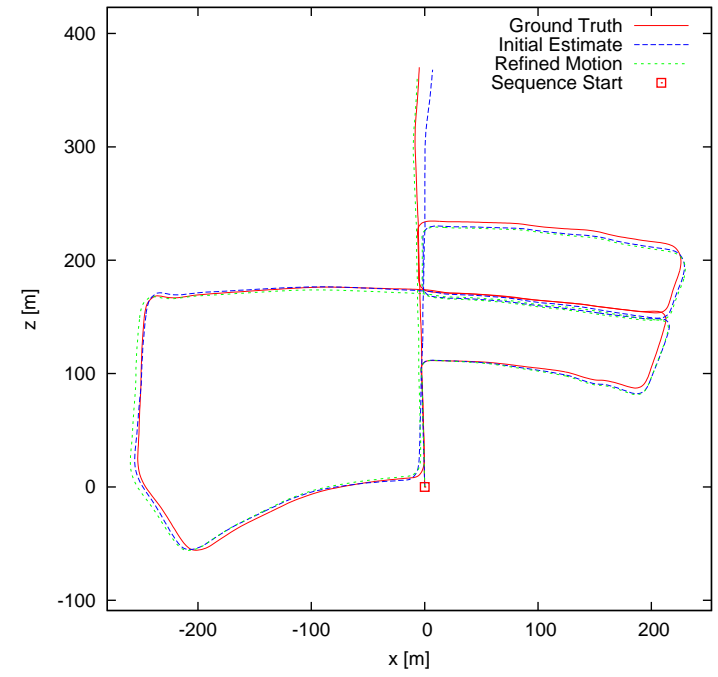

Fig. 13: Map built by our method (Initial Estimate and Refined Motion) vs. Ground Truth for the fifth sequence.

3) Asynchronous-to-Synchronous case: We also processed the results obtained by asynchronous method using the synchronous data processing algorithm. Basically, the camera poses in asynchronous case are obtained by using Equation (15). Starting from the obtained poses of the first set of cameras, we used Algorithm 2 under the assumption that Algorithm 1 synchronizes the camera pairs. Results obtained in each step for Fountain and Herz-Jesu sequences are shown in Table VII. Figure 14 shows cameras in the scene for one of the sequences. It can be observed that the camera poses obtained after the synchronous assumption are very satisfactory. However, they are not always as good as the ones obtained from asynchronous algorithm. This happens mainly because the synchronous algorithm is relatively more sensitive to the pose gaps. In few cases, when the asynchronous algorithm does not produce results very close to ground truth, the synchronous

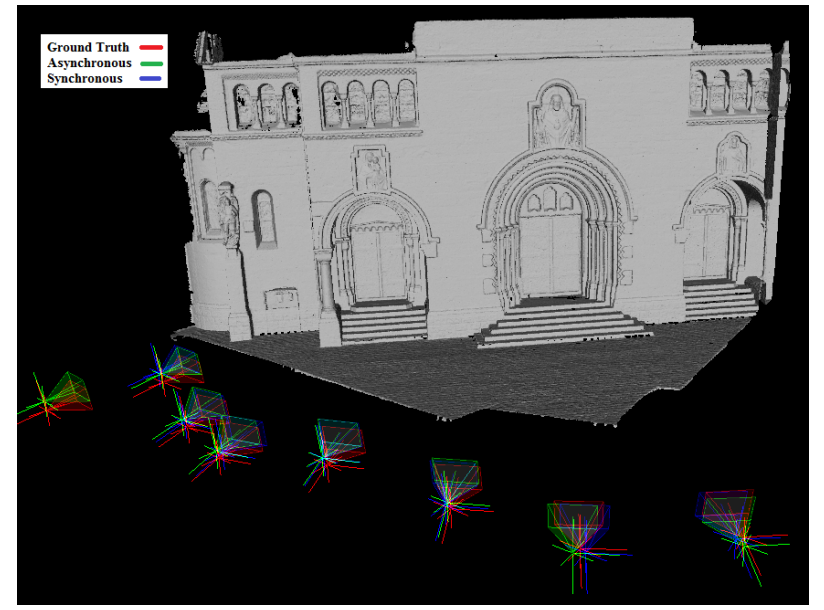

Fig. 14: Ground truth, Asynchronous-to-Synchronous Cameras poses in the scene.

\begin{tabular}{|c|c|c|c|c|}
\cline { 2 - 5 } \multicolumn{1}{c|}{} & \multicolumn{2}{c|}{ Asynchronous } & \multicolumn{2}{c|}{ Synchronous } \\
\cline { 2 - 5 } \multicolumn{1}{c|}{} & $\Delta R$ (mean) & $\Delta t($ mean $)$ & $\Delta R($ mean $)$ & $\Delta t$ (mean) \\
\hline Fountain-P11 & 0.0214 & 0.0074 & 0.0230 & 0.0111 \\
\hline Herz-Jesu & 0.0222 & 0.0182 & 0.0196 & 0.0191 \\
\hline
\end{tabular}

TABLE VII: Error measured during Asynchronous-toSynchronous case.

algorithm rather deteriorates the results instead of further improvement. Nevertheless, the absolute poses obtained from the asynchronous algorithm remains unaffected.

\section{CONCLUSION}

In this paper, we have proposed an optimization framework to accurately localize two or more cameras in a known environment. We have demonstrated the possibility of precisely registering $2 \mathrm{D}$ images to $3 \mathrm{D}$ scene using only feature points. Usage of a known 3D scene to refine the camera pose is key to achieve such accuracy. To make it possible, a direct 2D-to-3D registration method has also been integrated in the 
optimization process. When the 3D scene is known, even up to some inaccuracies, it is better to use this information for the refinement of the SfM reconstruction than using generalpurpose techniques such as BA.

\section{REFERENCES}

[1] D. Holz, C. Lorken, and H. Surmann, Continuous 3D sensing for navigation and SLAM in cluttered and dynamic environments, ICIF, 2008.

[2] Sattler, Torsten and Leibe, Bastian and Kobbelt, Leif, Fast image-based localization using direct 2D-to-3D matching, ICCV, 2011.

[3] Knopp, Jan and Sivic, Josef and Pajdla, Tomas, Avoiding confusing features in place recognition, ECCV, 2010.

[4] Tamaazousti, M. and Gay-Bellile, V. and Collette, S. N. and Bourgeois, S. and Dhome, M., NonLinear refinement of structure from motion reconstruction by taking advantage of a partial knowledge of the environment, CVPR, 2011.

[5] Christy, Stéphane and Horaud, Radu, Iterative pose computation from line correspondences, Comput. Vis. Image Underst., January, 1999, 137 144.

[6] Prasad, M. and Fitzgibbon, A. W. and Zisserman, A. and Van Gool, L., Finding Nemo: Deformable Object Class Modelling using Curve Matching, CVPR, 2010

[7] Liu, Lingyun and Stamos, Ioannis, Automatic 3D to 2D Registration for the Photorealistic Rendering of Urban Scenes, CVPR, 2005.

[8] Srikumar Ramalingam and Sofien Bouaziz and Peter Sturm and Matthew Brand, Geolocalization using Skylines from Omni-Images, ICCV Workshops, 2009.

[9] Corsini, M. and Dellepiane, M. and Ganovelli, F. and Gherardi, R. and Fusiello, A. and Scopigno, R., Fully Automatic Registration of Image Sets on Approximate Geometry, Int. J. Comput. Vision, March, 2013, 91-111.

[10] Taneja, Aparna and Ballan, Luca and Pollefeys, Marc, 3DIMPVT, Registration of Spherical Panoramic Images with Cadastral 3D Models, 2012.

[11] Viola, Paul and Wells,III, William M., Alignment by Maximization of Mutual Information, Int. J. Comput. Vision, September, 1997, 137-154.

[12] Clarkson, Matthew J. and Rueckert, Daniel and Hill, Derek L.G. and Hawkes, David J., Using Photo-Consistency to Register 2D Optical Images of the Human Face to a 3D Surface Model, IEEE Trans. Pattern Anal. Mach. Intell., November, 2001, 1266-1280.

[13] Nistér, David, An Efficient Solution to the Five-Point Relative Pose Problem, IEEE Trans. Pattern Anal. Mach. Intell., June, 2004, 756-777.

[14] Prasad, M. and Fitzgibbon, A. W. and Zisserman, A. and Van Gool, L., Finding Nemo: Deformable Object Class Modelling using Curve Matching, CVPR, 2010.

[15] Fitzgibbon, A., Robust registration of 2D and 3D point sets, Image and Vision Computing, December, 2003, 1145-1153.

[16] Martin, K. and Jakob W., Iterative Closest Point, Technical University of Denmark, 2012.

[17] Lowe, David G., Distinctive Image Features from Scale-Invariant Keypoints, Int. J. Comput. Vision, November, 2004, 91-110.

[18] Joel A. Hesch and Stergios I. Roumeliotis, A Direct Least-Squares (DLS) Method for PnP, ICCV, 2011.

[19] J.W. Weingarten, G. Gruener, and R. Siegwart, A state-of-the-art 3D sensor for robot navigation, IROS, 2004.

[20] B. Williams, M. Cummins, J. Neira, P. Newman, I. Reid, and J. Tardós, A comparison of loop closing techniques in monocular SLAM, Robot. Auton. Syst., 2009.

[21] Alice E. Smith and David W. Coit, Penalty Functions, University of Pittsburgh, September 1995.

[22] Y. Taguchi, and Y.D. Jian, S. Ramalingam, and C. Feng, Point-Plane SLAM for Hand-Held 3D Sensors, ICRA, 2013.

[23] A.J.B. Trevor, J.G. Rogers, and H.I. Christensen, Planar surface SLAM with 3D and 2D sensors, ICRA, 2012.

[24] Y. Bok, Y. Jeong, D.G. Choi, and I.S. Kweon, Capturing Village-leve Heritages with a Hand-held Camera-Laser Fusion Sensor, Int. J. Comput. Vision, 2011.

[25] D. Nister, A minimal solution to the generalised 3-point pose problem, CVPR, 2004.

[26] M. Lhuillier, Incremental Fusion of Structure-from-Motion and GPS Using Constrained Bundle Adjustments, IEEE Trans. Pattern Anal. Mach. Intell., 2012.

[27] M.I. A. Lourakis and A.A. Argyros, SBA: A Software Package for Generic Sparse Bundle Adjustment, ACM Trans. Math. Software, 2009
[28] A. Chiuso, P. Favaro, H. Jin, and S. Soatto, 3-D motion and structure from 2-D motion causally integrated over time: Implementation, ECCV, 2000 .

[29] D. Nister, O. Naroditsky, and J. Bergen, Visual odometry, CVPR, 2004.

[30] D. Nister, An efficient solution to the five-point relative pose problem, IEEE Trans. Pattern Anal. Mach. Intell., 2004

[31] R. Koch, Dynamic 3-d scene analysis through synthesis feedback control, IEEE Trans. Pattern Anal. Mach. Intell., 1993.

[32] A. Comport, E. Malis, and P. Rives, Accurate Quadri-focal Tracking for Robust 3D Visual Odometry, ICRA, 2007.

[33] P.J. Besl, and N.D. McKay, A method for registration of 3-D shapes, IEEE Trans. Pattern Anal. Mach. Intell., 1992.

[34] S. Rusinkiewicz, and M. Levoy, Efficient variants of the ICP algo-rithm, 3DIM, 2001.

[35] C. Kerl, J. Sturm, and D. Cremers, Dense Visual SLAM for RGB-D Cameras, IROS, 2013.

[36] P. Henry, M. Krainin, E. Herbst, X. Ren, and D. Fox, RGB-D Mapping: Using Kinect-Style Depth Cameras for Dense 3D Modeling of Indoor Environments, IJRR, 2012.

[37] R.A. Newcombe, S. Izadi, O. Hilliges, D. Molyneaux, D. Kim, A.J. Davison, P. Kohli, J. Shotton, S. Hodges, and A. Fitzgibbon, KinectFusion: Real-time Dense Surface Mapping and Tracking, ISMAR, 2011.

[38] A. Nüchter, K. Lingemann, J. Hertzberg, and H. Surmann, 6D SLAM 3D Mapping Outdoor Environments: Research Articles, J. Field Robot., 2007.

[39] B. Triggs, P. Mclauchlan, R. Hartley, and A. Fitzgibbon, Bundle adjustment - a modern synthesis, Vision Algorithms: Theory and Practice, LNCS, 2000.

[40] A. Geiger, P. Lenz, C. Stiller, and R. Urtasun, Vision meets Robotics: The KITTI Dataset, International Journal of Robotics Research, 2013.

[41] D. P. Paudel, C. Demonceaux, A. Habed, and P. Vasseur, Localization of 2D Cameras in a Known Environment using Direct 2D-3D Registration, ICPR, 2014

[42] D. P. Paudel, C. Demonceaux, A. Habed, P. Vasseur, and I. S. Kweon, 2D-3D Camera Fusion for Visual Odometry in Outdoor Environments, IROS, 2014

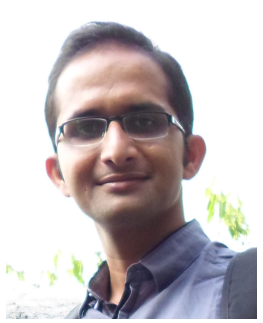

Danda Pani Paudel received Master's degree in Computer Vision from Université de Bourgogne, France in 2012. He is currently a Ph.D. candidate at Le2i UMR CNRS, Université de Bourgogne. His research interest include visual SLAM, Computer Vision, and Global Optimization.

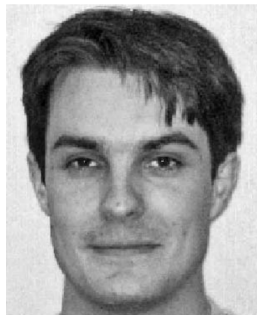

Cédric Demonceaux received the MS degree in Mathematics in 2001 and the Ph.D. degree in Image Processing from the Université de Picardie Jules Verne (UPJV), France, in 2004. In 2005, he became an Associate Professor at MIS-UPJV. Since 2010 he has been a CNRS-Higher Education chair at Le2i UMR CNRS, Université de Bourgogne. His research interests are in Image Processing, Computer Vision, and robotics. 




Adlane Habed is an Associate Professor of Computer Science at the University of Strasbourg (France) and a member of the ICube (CNRS) research laboratory. He received a Ph.D. in Computer Science from the University of Sherbrooke (Canada) in 2005. From 2001 to 2007, he served as a Computer Science Full-time Lecturer (2001 2005) and as an Assistant Professor (2005 - 2007) at the University of Windsor's School of Computer Science (Canada). Prior to his current appointment, he held an Assistant Professor position, from 2007 to 2012, at the University of Bourgogne (France) and was member of the Le2i (CNRS) research laboratory. His research interests are in the field Computer Vision and Optimization.

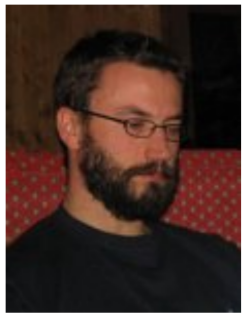

Pascal Vasseur received the MS degree in System Control from the Université de Technologie de Compiégne, France, in 1995, and the Ph.D. degree in Automatic Control from the Universite de Picardie Jules Verne, France, in 1998. He was an Associate Professor at the University Institute of Technology of Amiens between 1999 and 2010. He is now a professor at the University of Rouen in the LITIS Laboratory. His research interests include Computer Vision and its applications to mobile and aerial robots. 